\title{
MITTELALTERLICHER SÄBEL MIT EINER ARMENISCHEN INSCHRIFT, GEFUNDEN IM SUBPOLAREN URAL \\ VON
}

REPSIME DJANPOLADIAN / ANATOLIJ KIRPICNIKOV

DIE Geologen, die an den Osthängen des Gebirgsrückens Narodinski, am Ufer des Flusses Malaja Tynagota (Bezirk Berezovski, Oblast Tjumen) arbeiteten, bemerkten neben einer Lärche in dem vom Regen ausgewaschenen Boden einen aus der Erde ragenden Metallgegenstand, der sich als eine Säbelklinge erwies. Die von ihnen oberflächlich am Ort durchgeführte Suche ergab keine neuen Funde. Der die Geologen begleitende Journalist I. J. Titov sandte den versehentlich zerbrochenen Säbel zwecks Bestimmung und Untersuchung an die Leningrader Abteilung des Archäologischen Instituts der Akademie der Wissenschaften der UdSSR.

Wenn man vom frischen Bruch absieht, ist die Klinge verhältnismäßig gut erhalten, ihre Gesamtlänge mit Griff beträgt $1 \mathrm{~m}$, bei einer Breite von $3,5 \mathrm{~cm}$ und einer Dicke von $0,6 \mathrm{~cm}$. Ebenso ist die rautenförmige Parierstange erhalten, ihre Länge beträgt $8 \mathrm{~cm}$. Die Klinge ist leicht gekrümmt, das Ende ist zweischneidig. Die Achse des Griffangels, der offenbar früher Beschläge und einen Knauf trug, ist leicht zur Schneide geneigt (Bild 1). An der Übergangsstelle vom Griff zur Klinge ist eine Metallmanschette erhalten, die zur Befestigung der Parierstange dient.

Seinem Typ nach gehört der Säbel ins XII-XIII. Jh.; davon zeugen folgende Merkmale: leichte und gleichmäßige Krümmung der Klinge, rautenförmige Parierstange mit Haltemanschette, die Abmessungen der Klinge als auch ihre konstruktive Eigenart. ${ }^{1}$

Im oberen Teil der Klinge befindet sich ein $1 \mathrm{~cm}$ breiter und $20 \mathrm{~cm}$ langer Streifen, der mit Zeichen und einem Ornament versehen ist. In den Vertiefungen sind Spuren einer Vergoldung erhalten. Zu beiden Seiten der Klingenmarke befinden sich kleine, an den Enden verzweigende Kreuze. Der ornamentale Teil der Klingenmarke besteht aus einer Folge von 20 gestanzten Doppeldreiecken. Ihnen gehen 10 gravierte Zeichen voraus, die bei genauerer Betrachtung eine Ähnlichkeit mit Buchstaben des armenischen Alphabets aufwiesen (Bild 2).

${ }^{1}$ A. N. KinpičnIKov: Drevnerusskoje oružije. Vyp. I. Meči i sabli IX-XIII vv. Moskva-Leningrad 1966, S. 69 ff. 
Laut vorhandenen Angaben erschienen die Säbel in Eurasien im VII-VIII. Jh. in einem breiten Raum vom Altai bis rach Ungarn. An der Herstellung dieser Waffen nahmen asiatische Nomaden und diejeni-

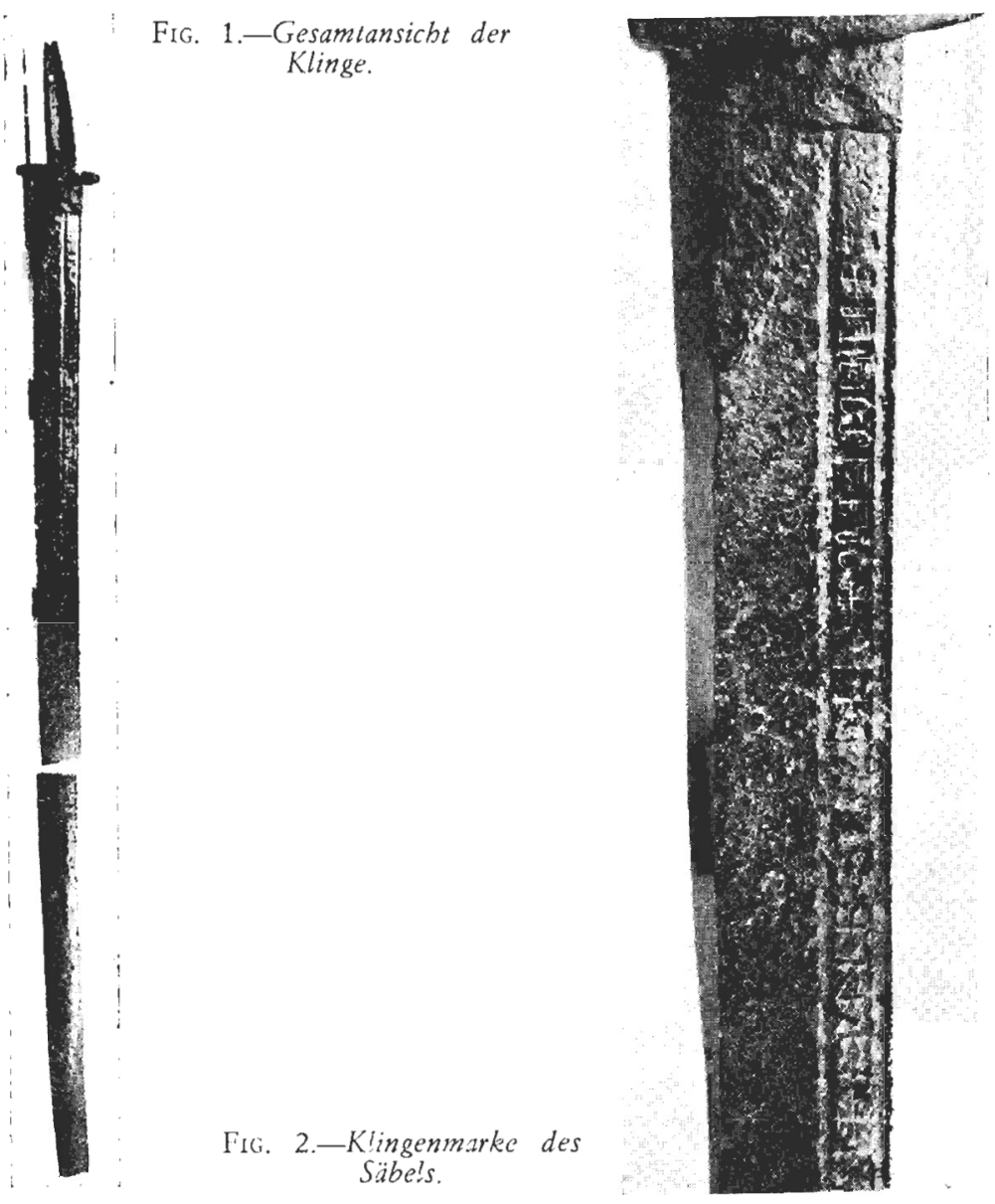

gen teil, die mit jenen gekämpft hatten. Nach Süd- und Zeniraleuropa dringt der Säbel um die Zeit der späten Kreuzzüge. Zu den erste- Staaten, die die gelsrümmte Klinge annahmen, gehörten Rußland und Ungarn. Es wird angenommen, daß weder Vorderasien noch Ägypten bis zum 
XIII. Jh. diese Waffe kannten. ${ }^{2}$ Das Verzeichnis der Länder, die recht früh von Schwertern zu Säbeln übergingen, muß noch ergänzt werden. Bevor wir uns in das Studium dieses Säbels vertiefen und seinen Ursprung festzustellen versuchen, müssen wir zunächst die Zeichen auf der Klingenmarke deuten; sollte es sich erweisen, daß sie eine Inschrift bilden, so ist ihre Entzifferung zu versuchen.

Bei den mittelalterlichen Handwerkern war es üblich, auf ihre Erzeugnisse entweder den Namen des Meisters oder einen wohlgemeinten Spruch bzw. sogar ein Gebet zu setzen. Der Wunsch, lange Inschriften auf einem relativ beschränkten Raum eines kleinen Erzeugnisses anzubringen, führte die Handwerker zur Schaffung von Ligaturen und Kryptogrammen. Es sind Fälle von kopfzerbrechenden Wörtern bekannt, die oft unenträtselt geblieben sind. Es gibt noch einen Umstand, der das Entziffern von alten Inschriften erschwert: Sie wurden geschrieben, umgeschrieben, von einem Gegenstand auf den anderen kopiert-dabei von Leuten, die die wahre Bedeutung der Inschriften bzw. Buchstaben nicht kannten (Gesellen, Lehrlinge). Das führte manchmal zu einer Entstellung der Inschrift, zu ihrer Verwandlung in ein Ornament.

Unter den Metallgegenständen war die Verzierung mit verschiedenen Inschriften besonders bei den Schwertern verbreitet. Im XII-XIII. Jh. wetteiferten die Meister sogar in der Länge und Ausdruckskraft der Inschriften; die gravierenden Meister gingen mitunter recht frei mit ihren Texten um, sie kürzten die Wörter, änderten die Buchstabenordnung, schrieben ein und dasselbe Wort auf verschiedene Art. Sogar in den verbreiteten Schwertinschriften sind stark verzerrte Wörter anzutreffen, deren Entzifferung nur durch Vergleich mit bereits entschlüsselten Mustern gelingt. Dieser Umstand erschwert öfters die Entzifferung eines Textes. ${ }^{3}$

Die Technik der beschrifteten Klingenmarken auf blanken Waffen, und zwar auf Schwertern, war stets mit der europäischen Tradition verbunden. Unter den z.Z. bekanntnen Säbeln des frühen Mittelalters sind fast keine mit einer Inschrift bekannt. Wir wissen nur von e in em Säbel des IX-X. Jh. mit einer arabischen wohlwünschenden Inschrift, der in Tuwa gefunden wurde. Beschriftete Säbel sind zum Unterschied von den Schwertern viel seltener und später (XVI. Jh.) anzutreffen. Dieser Umstand machte ebenfalls die Entzifferung der Inschrift auf dem Uralsäbel besonders wichtig.

Obwohl die Klinge relativ gut erhalten ist, war die Inschrift mit teils

2 Ibid., S. 61.

'A. N. KıRpıčnıKov: Nadpisi $i$ znaki na klinkach vostočnojevropejskich mečej IX-XIII vv. Skandinavskij sbornik, t. XI. Tallin 1966, S. 249-294. 
verwischter Vergoldung von einer Rostschicht bedeckt und deshalb unklar. Es wurden technische Mittel angewandt, um diese Schicht zu entfernen, die Klinge wurde vorsichtig gesäubert, doch wegen den Beschädigungen, die den Zeichen selber innehafteten, blieb die Entzifferung dennoch schwierig. Eine

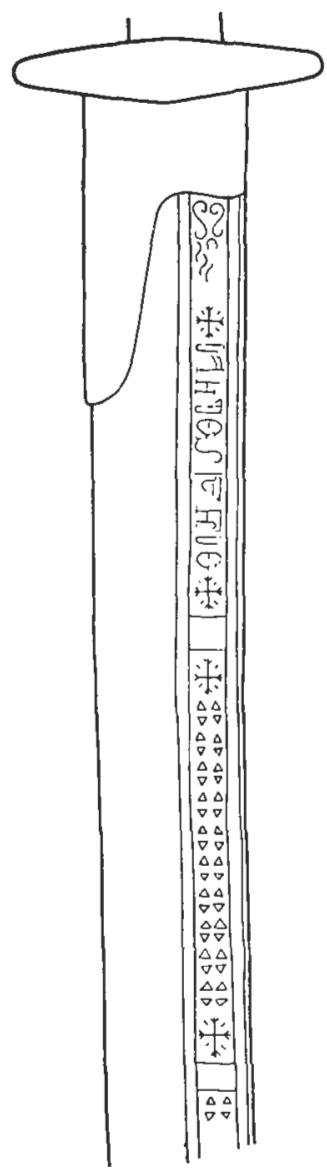

FIg. 3.-Durcbzeichnung der Klingenmarke. wesentliche Hilfe erwies uns das Leningrader Forschungslaboratorium für Gerichtsexpertise. Durch den Vergleich des von den Kriminalisten erhaltenen Bildes mit den von uns vielfach durchgezeichneten Buchstaben gelang es, den größten Teil der Inschrift zu restaurieren (Bild 3).

Die $8 \mathrm{~cm}$ lange Inschrift auf unserem Säbel ist fast vollständig erhalten; sie besteht aus 10 gravierten Zeichen, die von den beiden abschließenden Kreuzen begrenzt ist.

Von den 10 Zeichen scheinen 8 Buchstaben des armenischen Alphabets zu sein. Das erste Zeichen ist unklar und deshalb zweifelhaft. Es erinnert an den Buchstaben $\mathrm{I}_{\mathrm{I}}(\mathrm{K})$ (hier und weiter wird in Klammern die bibliothekarische Transkription angeführt); führt man jedoch den ersten vertikalen Strich zu Ende, so kann man es auch wie $U(S)$ lesen. Es sind auch andere Varianten vorhanden, die weiter unten besprochen werden.

Das zweite und dritte Zeichen gleichen keinem einzigen armenischen Buchstaben und lassen sich nicht entziffern. Die nächsten sieben Zeichen sind deutlicher abgebildet und lassen sich mit Buchstaben des armenischen Alphabets identifizieren. Im vierten Zeichen ist ein 'l $(\mathrm{P}) \mathrm{zu}$ ersehen, dessen obere Gabelung mit einem dünnen Goldstrich versehen war und kaum zu erkennen ist. Weiter kommt der Buchstabe l; (E), dann $\$(\mathrm{~T})$, sodann $\mathrm{IU}(\mathrm{CH})$, es folgt eine Ligatur der Buchstaben $S$ und $P(T R)$. Die letzten zwei Zeichen kann man wie $\mathrm{l},(\mathrm{N})$ und $\mathrm{L}(\mathrm{E})$ lesen.

Die Enden des vierten, fünften und zehnten Buchstaben sind nach innen gewandt und haben ergänzende Endstriche. Über dem siebenten und achten Buchstaben sind waagerechte Striche vorhanden, die man offenbar als Abkürzungszeichen ansehen kann, welche besagen, daß diese Zeichen 
nicht als einzelne Buchstaben, sondern als Kurzwörter bzw. _- silben anzusehen sind. Das gibt uns die Möglichkeit, sie voll zu lesen, und zwar das Zeichen /1s unter dem Abkürzungsstrich als Wort Jul!? (CHAC). Solch eine Kurzschriftform dieses Wortes ist bei armenischen Inschriften auf Steinen gebräuchlich und oft anzutreffen, sie kommt auch in $\mathrm{Ma}$ nuskripten vor.

Die achte Ligatur $\left.\$\right|^{\prime}$, die ebenfalls unter einem Abkürzungszeichen steht und keinen Vokal enthält, kann als Wort SLT (TER) oder als Silbe SIflY' (TUR) gelesen werden. Die letztere Möglichkeit ist zu bevorzugen, sie gibt zusammen mit dem vorhergehenden Zeichen luL? (CHAC) und dem Bindezeichen l" (A) den armenischen Männernamen JULPUSOF" (CHACATUR). Weitere Beobachtungen an der Inschrift bestätigen die Wahrscheinlichkeit einer solchen Lesart dieser Ligaturen. Der obenerwähnte Männername ist im armenischen Milieu seit dem XI. Jh. und bis auf den heutigen Tag verbreitet. Dieser Männername auf dem Säbel sollte wahrscheinlich auf den Besitzer oder Hersteller hinweisen.

Vor den Namen wird in einer Inschrift gewöhnlich der Titel, Rang oder Beruf des Menschen gesetzt. Um zu klären, wer dieser CHACATUR gewesen war, müssen wir uns jetzt dem vierten, fünften und sechsten Zeichen zuwenden, die man als Silbe "il;s (PET) lesen kann, was die Endung des Wortes IU("I) 'S (VARPET) zu sein scheint, das ebenfalls aus sechs Buchstaben besteht. Leider haben das zweite und dritte Zeichen ihre ursprüngliche Form verloren und sind, wie schon gesagt, mit keinem armenischen Buchstaben in Verbindung zu setzen. Es wurde schon erwähnt, daß erstes Zeichen als $4(K)$ oder $U(S)$ gelesen werden kann; wenn man jedoch eine graphische Eigenheit der Zeichen unserer Inschrift berücksichtigt, daß nämlich z.B. beim vierten, fünften und zehnten Zeichen die Enden nach innen gerundet sind, so kann man, wenn auch mit großem Vorbehalt, das erste Zeichen mit $\%$. (V) identifizieren, wobei der Meister den Endstrich des Buchstaben nicht nach rechts, wie üblich, geführt, sondern nach links und oben abgerundet hat. Das entspräche dann dem ersten Buchstaben des Wortes $1.14 \%$ T) (VARPET).

Endgültig erhält dann die Inschrift auf der Klinge den Wortlaut:

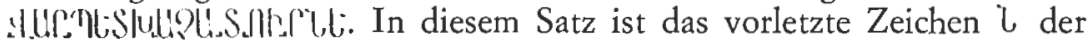
bestimmte Artikel, das Zeichen $l$ aber, daß das fünfte Zeichen wiederholt, ${ }^{4}$ ist eine ungenaue Wiedergabe eines Hilfsverbs in der 3. Pers. Sing. (ist).

Wir nehmen also an, daß die Inschrift auf der Klinge mitteilt, dieser Säbel sei von varpet-Chačatur angefertigt worden. Wir haben es folglich

4 Es müßte in diesem Falle das Zeichen I: $(\dot{E})$ gesetzt werden, das ähnlich gesprochen und geschrieben wird. 
nicht mit einer besitzanzeigenden oder beschwörenden, sondern mit einer betrieblichen Warenmarke zu tun.

Das Wort «varpet» bedeutete im mittelalterlichen Armenien nicht bloß einen Meister seines Faches. Es war faktisch der Titel eines Handwerkers, den er nach Ablegen einer bestimmten Prüfung erhielt. Dieser Titel berechtigte ihn, eine eigene Werkstatt und eigene Schüler zu haben. Offenbar ist also die Inschrift als eine Art Warenzeichen der Werkstatt eines vielleicht seinerzeit bekanntnen Waffenmeisters Chačatur anzusehen.

$$
* * *
$$

Nach der Entzifferung der Inschrift müssen wir uns jetzt den Umständen und Wegen zưwenden, dank denen der Säbel nach dem Nordural gelangen konnte.

Aus den arabischen geographischen Schriften des IX-XIII. Jh. (IbnFadlan, al-Garnati, al-Biruni, Marvazi, Jakut, Ibn Batuta, Kazvini u.a.) erfahren wir, daß im weiten Nordgelände am Meer des Nebels oder des Dämmerlichts (so nannten sie das Nordpolarmeer) das Volk Juru oder laut den russischen Chroniken "Jugra» wohnte. ${ }^{5}$ Es wird als wildes, düsteres Volk geschildert, das hauptsächlich von Jagd und Fischfang existierte. Es unterhielt Handelsbeziehungen mit den Wolgabulgaren, bei denen es seine Edelpelzfelle —Feh, Hermelin, Zobel— als auch Sklaven gegen Kleidung, Salz und verschiedene andere Waren eintauschte, darunter -wie von Zeitgenossen besonders unterstrichen wird- auch Schwerter.

Der arabische Geograph und Reisende des XII. Jh. Abu Hamid alGarnati, der 1150-1153 bei den Wolgabulgaren weilte, schreibt darüber: «Diese Schwerter aber, die aus den Islamländern zu den Bulgaren eingeführt werden, bringen einen großen Gewinn. Die Bulgaren bringen sie ihrerseits nach Visu (entspricht dem «Ves'» der russischen ChronikenAnm. der Verfasser), wo es viele Biber gibt, die Visu aber bringen sie nach Juru und tauschen sie bei den Einwohnern gegen Zobelfelle, Sklaven und Sklavinnen ein». ${ }^{6}$

An einer anderen Stelle heißt es bei ihm: «Und die Menschen bringen aus den Islamländern Schwerter, die in Zanjan, Abhar, Tabriz und Esfahan (Nordiran) hergestellt werden, in Form von Klingen, ohne Griff und Verzierungen, als bloßes Eisen, wie es aus dem Feuer kommt. Diese

${ }^{5}$ B. N. ZACHoder: Kaspijskij svod svedenij o Vostočnoj Evrope, t. II. Moskva 1967, S. 59-69.

- Abu Hamid al-Andalusi (oder al-Garnati): Tubfat al-albäb. Ed. César E. Dubler. Madrid 1953, S. 18 (die Übersetzung dieser Stelle verdanken wir O. G. Bol'šakov). 
Schwerter werden stark gehärtet, so daß wenn man ein Schwert an einem Faden aufhängt und mit dem Fingernagel oder einem Eisen --bzw. Holzstück anschlägt, ein lange andauernder Klang ertönt. Und diese Schwerter sind eben diejenigen, die sich für die Einfuhr nach Juru eignen». ${ }^{7}$

Die Bewohner des Nordens waren keine kriegerischen Völker, die Schwerter und Säbel wurden von ihnen nicht für den Krieg, sondern als Metall angeschafft, aus dem sie Messer, Harpunen und andere Gebrauchsgegenstände anfertigten. Außerdem dienten die Schwerter bei ihnen auch für verschiedene Bräuche. «Man sagt», schreibt al-Garnati, «daß wenn die Juru (Jugra) keine Schwerter ins Wasser werfen, sie dann keine Fische fangen und Hunger leiden werden». ${ }^{8}$

Über den lebhaften Handel der Bulgaren mit den Nordvölkerschaften schreiben auch spätere arabische und persische Autoren. Statt von eingeführten Schwertern sprechen sie von Säbeln (der Perser Ajufi, vor 1236) ${ }^{9}$ und beschreiben ausführlich sowohl die Fortbewegung auf Schlitten mit vorgespannten Hunden, als auch die Begleiter auf Schneeschuhen.

Für das geschichtliche Studium unseres Säbels ist ein anderer Bericht von al-Garnati von erheblichem Interesse - über die Bräuche und Sitten der arktischen Völker. Den arabischen Reisenden setzte in Erstaunen die Art des von ihnen betriebenen Tauschhandels, Er schreibt: «Jeder Händler bringt seine Waren gesondert, mit einem eigenen Zeichen versehen; dann ziehen sie sich zurück, und wenn sie zurückkehren, so finden sie andere, für ihr Land nötige Waren. Jeder findet etwas neben den von ihn hingelegten Stücken; paßt es ihm, so nimmt er es an sich, widrigenfalls nimmt er die von ihm gebrachten Waren zurück...». ${ }^{10}$

So ging dieser stumme, des öfteren vielfache Tausch vor sich, der scheinbar an bestimmten Stellen stattfand. Vergleicht man diesen Bericht mit den Begleitumständen unseres Säbelfundes, so kommt der Gedanke, ob die Klinge nicht auf solch einem Tauschort vergessen worden sei. Da Käufer und Verkäufer, wie es scheint, mehrmals abwechselnd ihren Platz einnahmen, ist der Verlust von Gegenständen nicht ausgeschlossen.

Es ist kaum anzunehmen, daß der Säbel während eines Kriegszugs verloren ging - die Jugra waren ein friedliches Volk, das weder Rosse noch den Säbelkampf kannte. Die Klinge konnte auch nicht als untauglich fortgeworfen worden sein - die Schneide weist keine Scharten auf, sie sieht geschärft aus, schien also vor einigen Hundert Jahren voll-

7 Ibid., S. 15-16, Übersetzung von O. G. Bol'šakov.

${ }^{8}$ A. L. Mongajt: Abu Chamid al-Garnati $i$ ego putešestvie $v$ russkie zemli 1150-1153 gg, «Istorija SSSR», 1959, I, S. 173.

- J. Marquart: Ein arabischer Bericht über die arktischen Länder, in: Ungarisches Jabrbuch, t. IV, 1924, S. 314.

${ }_{10}$ A. L. Mongajt, 1. c., S. 172. 
kommen neu gewesen zu sein. Die von uns vorgeschlagene Version, die Klinge sei am Tauschort verloren, ist natürlich nicht die einzig mögliche - sie kann unterwegs beim Überschreiten eines Passes verloren worden sein, vielleicht auch zu einem Begräbnis gehört haben. Wie dem auch sei, wir haben es mit einem seltenen transkaukasischen technischen Erzeugnis zu tun, das viele hundert Kilometer von seiner Heimat auftauchte. Die Klinge konnte in den Nordural im Altertum gelangt sein -beim Tauschhandel der bulgarischen Kaufleute mit den arktischen Völkern.

Diesem Schluß widerspricht auch die Fertigungstechnik des Säbels nicht. Seine metallographische Untersuchung wurde am Lehrstuhl für Metallkunde des Leningrader Polytechnischen Instituts durchgeführt. Es erwies sich, daß die Klinge aus einem aufgekohlten Rohling geschmiedet wurde. Beim Erhitzen vor dem Härten kam es zu einer Oberflächenentkohlung. Als Ergebnis entstand eine dreischichtige Struktur der Klinge, die aus zwei äußeren und einer inneren Schicht bestand. Dadurch wurde die für ein Qualitätserzeugnis benötigte Kombination von Zähigkeit und Härte erreicht. Sodann wurde die gehärtete Klinge geschärft, wobei auf der Oberfläche als Schneide der Stahlkern erschien. Dank der sachgemäßen Wärmebehandlung wurde die scharfe Schneidenkante besonders hart.

Wie wir sehen, war es keine Damaszener Ganzstahlklinge, sondern zeichnete sich durch eine besondere Härtungsart aus, wovon eben unsere mittelalterlichen Quellen sprechen. Denken wir daran, daß die aus Tiegelgußstahl angefertigten Klingen der Ostländer im Norden keinen Anklang fanden, da sie vom Frost brüchig wurden und in Stücke gingen.

Wie schon oben erwähnt, wiesen die arabischen Authoren mehrmals darauf hin, daß die von den nördlichen Völkern gern erworbenen Schwerter in den Islamländern angefertigt wurden. Nunmehr erfahren wir aber, daß auch im Transkaukasus Waffen angefertigt wurden, die den Norden erreichten.

Der in der Oblast Tjumen gefundene Säbel ist die erste Illustration zu den Mitteilungen der arabischen Quellen über die Lieferung von Klingen nach Jugra, doch stammt er nicht aus muslimischem, sondern aus christlichen Milieu, wovon außer der armenischen Inschrift und dem christlichen Namen des Meisters auch die vier auf der Klingenmarke untergebrachten kleinen Kreuze sprechen.

Daß Waffenschmieden im alten Armenien existierten, ist aus Literaturquellen bekannt, die öfters vom weitverbreiteten Waffenhandwerk berichten. ${ }^{11}$

"B. N. Arakeljan: Goroda i remesla Armenii v IX-XIII vv, t. I. Erevan 1958, S. 142 (in arm. Spr.). 
Der Säbel aus dem XII-XIII. Jh., gefunden im Nordural und offensichtlich in Armenien hergestellt, ist z.Z. ein einmaliges Muster von armenischen Waffen dieser Art. Es ist möglich, daß dieses Erzeugnis der transkaukasischen Waffenkunst über die armenische Handelskolonie bei den Wolgabulgaren nach dem Norden gelangte.

Im Subpolaren Ural ist also der in Eurasien älteste Säbel gefunden worden, der mit einer Klingenmarke versehen ist. In waffengeschichtlicher Hinsicht hat diese Tatsache sowohl nationale als auch internationale Bedeutung; hoffentlich wird sie die entsprechenden Fachleute dazu anregen, solche Säbelinschriften zu suchen und zu säubern. Etwas Ähnliches geschah doch mit den mittelalterlichen Schwertern, von denen wir erfahren haben, daß drei von je vier Stücken Inschriften und Verzierungen besaßen. 